\title{
Hommage à la diversité
}

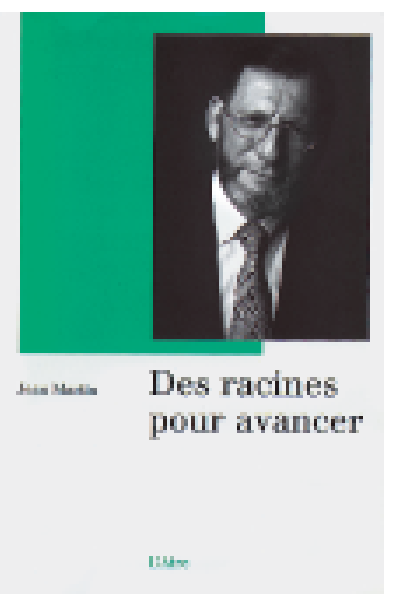

Jean Martin. Des racines pour avancer. Vevey: Editions de l'Aire; 2005. 327 pages. ISBN: 2-88108-752-3.
Au Moyen Age, il existait encore: l'homme universel qui possédait une connaissance holistique de son temps et jouissait d'une grande notoriété. Il y a longtemps que cette époque est révolue. La multiplication exponentielle du savoir des temps modernes conduit forcément à la spécialisation, accompagnée, peut-être pas par hasard, d'une diminution du prestige d'un savoir plus général. En ce qui concerne la médecine, le patient ne sait bientôt plus à quel saint se vouer: s'il fait confiance au spécialiste d'un domaine et recourt volontiers à son traitement, il se plaindra cependant de son point de vue étroit ou chez un jeune confrère, de son manque d'expérience de la vie. Reste à prouver que c'est toujours le cas.

Sur cette toile de fond, le contre-exemple est intéressant: le médecin qui se signale par la vastitude de son savoir et jouit néanmoins d'une grande considération. Jean Martin, ancien médecin cantonal vaudois, privat-docent, journaliste et notamment membre du conseil d'administration des Editions médicales suisses, appartient certainement à cette catégorie. On peut s'en convaincre par son dernier ouvrage «Des racines pour avancer» ${ }^{*}$, où il captive son lectorat par son parcours et sa vision du monde.

De ses racines dans le milieu viticole vaudois et d'une famille chrétienne engagée, il a hérité de valeurs fondamentales auxquelles il est resté fidèle tout en cultivant une grande ouverture d'esprit et une curiosité du monde extérieur. Cette notion de «monde» doit être comprise chez lui comme universelle. Le nombre de pays et de régions qu'il a visités est impressionnant et ses connaissances dépassent la simple expérience touristique. Il a travaillé notamment dans la «Selva» péruvienne, en Inde et au Cameroun, où ses séjours ont parfois duré plusieurs années. Ce n'est pas par hasard qu'il préface un chapitre avec une citation d'Aristophane: «Où l'on est bien, là est la patrie.»
Mais Jean Martin a aussi abordé avec passion le monde de l'art en général et de la littérature en particulier, de la philosophie et de la théorie de la science. Son intérêt à cet égard a débouché sur un engagement sans faille pour les questions d'éthique, en sa qualité, notamment, de membre de la Commission nationale d'éthique pour la médecine humaine. Le lien à la pratique et à la terre est une de ses caractéristiques, lui qui s'est penché professionnellement et en tant que parlementaire sur les problèmes et intérêts de la population de son canton d'origine.

S'il s'attribue lui-même la mentalité d'un «coureur de fond», c'est à prendre aussi au sens propre. Il n'aurait pas pu, sinon, assumer le nombre de tâches qu'il a accomplies et accomplit encore. Après tout ce qui vient d'être dit, nul ne s'étonnera qu'il n'ait pas choisi cette formule seulement comme métaphore. En effet, Jean Martin a réellement fait de la course à pied. Preuve en est sa participation au marathon de New York, aux $100 \mathrm{~km}$ de Bienne et au parcours de montagne Sierre-Zinal. L'endurance et la ténacité montrées dans son travail quotidien, en tant que politique et médecin cantonal, devraient donner force à son credo: «Rendre possible le nécessaire.»

Il est vain de vouloir ici répertorier toutes les facettes du personnage, que l'on retrouve dans son «autoportrait». Le choix de rassembler de petits articles et des essais dans un livre de plus de 300 pages donne l'avantage au lecteur de piquer au hasard et selon ses envies tel ou tel passage pour obtenir une riche image d'ensemble. Mais l'inconvénient pourrait être aussi de manquer un élément du puzzle. N'ayons crainte cependant: cette forme d'écriture convient parfaitement à un auteur qui utilise ses racines pour avancer.

Bruno Kesseli 